\title{
Absence of dual fluorescence with 4-( dimethylamino) phenylacetylene. A comparison between experimental results and theoretical predictions
}

\author{
Klaas A. Zachariasse, Mauricio Grobys, Erich Tauer \\ Max-Planck-Institut für biophysikalische Chemie, Spektroskopie und Photochemische Kinetik, D-37070 Göttingen, Germany
}

Received 21 April 1997; in final form 5 June 1997

\begin{abstract}
From ab initio electronic structure calculations it has been predicted that 4-(dimethylamino)phenylacetylene (DACET) will undergo an exothermic intramolecular charge transfer (ICT) reaction with dual fluorescence even under isolated-molecule conditions. Photostationary and time-resolved fluorescence measurements reveal however that an ICT reaction does not occur with DACET under any condition of solvent polarity or temperature studied. DACET is similar to 4-(methyl)aminobenzonitrile, which shows only fluorescence from a locally excited state, but is clearly different from the dual fluorescent 4-(dimethyl)aminobenzonitrile (DMABN). The energy gap $\triangle E\left(S_{1}, S_{2}\right)$ of DACET is larger than that of DMABN, supporting the importance of this gap in these ICT reactions. (C) 1997 Published by Elsevier Science B.V.
\end{abstract}

\section{Introduction}

Recently, Sobolewski and Domcke reported on ab initio electronic structure calculations with 4 -(dimethyl)aminobenzonitrile (DMABN) [1,2] and 4-(dimethylamino)phenylacetylene (DACET) [2]. They predicted that intramolecular charge transfer (ICT) would occur with DACET in the singlet excited state and that this molecule would show dual fluorescence even under isolated molecule conditions. It should be noted that in the case of the well-known dual fluorescent molecule DMABN [3-7] such an emission from a locally excited (LE) and a charge transfer (CT) state has not been observed in molecular jets [8].<smiles>CN(C)C1CCC(C#N)CC1</smiles>

DMABN<smiles>C#CC1CCC(C)CC1</smiles>

DACET

Over the years, a substantial number of calculations of the excited state properties of DMABN have been published, see Refs. [1,2,9,10] and references cited there. Whereas in the experimental studies a variety of excited state mechanisms have been proposed and discussed [3-7,11-13], involving different reaction pathways and reaction coordinates, the 
theoretical approaches took into account mainly one reaction coordinate in their description of the ICT reaction from the $\mathrm{LE}$ to the $\mathrm{CT}$ state. This reaction coordinate was taken to be the twisting of the dimethylamino group with respect to the benzonitrile moiety, from a planar starting configuration in the $S_{0}$ ground state to a perpendicular configuration of the final CT state: the 'twisted intramolecular charge transfer' or TICT mechanism [3,4]. All other configurational aspects of the molecule were generally left unchanged. In the DMABN calculations of SerranoAndrés et al. [9] the same bond distances were taken for the ground as well as for the LE and CT states. These authors calculated the excitation energies as a function of the twist angle of the amino group with respect to the benzonitrile plane and also for one additional value of the amino wagging angle. Note that the TICT hypothesis in its essence consists of a statement on the molecular structure of the final equilibrated CT state and that the detailed dynamics of the ICT reaction is not an integral part of this model [14].

In the calculations of Sobolewski and Domcke $[1,2]$, a change in the configuration of the cyano group of DMABN was taken into account as a second reaction coordinate next to the twisting of the dimethylamino group. It was concluded that the structure of the cyano group of DMABN changes by rehybridisation, from linear in the LE state to bent in the final CT state. For this CT state having an overall planar configuration with a bent cyano group, the term RICT ( $\mathrm{R}$ from rehybridised) was introduced. Bending of the cyano group rather than twisting of the amino group was suggested to be responsible for the stabilisation of the CT state in DMABN [1]. The calculations were carried out in three steps. First, the geometry of the electronic ground state and the TICT and RICT excited states were optimised by using HF/SCF and CIS methods $[1,2]$. The bond distances for the LE state were taken to be the same as for $S_{0}$. In the second stage, the CASSCF method [9] was employed at the fixed geometries obtained in the first step, followed by an inclusion of dynamic electron correlation effects via CASPT2, see Refs. $[1,2]$.

Similar calculations were performed with DACET [2], a molecule which is isoelectronic with DMABN. As a result, an energetically low-lying CT state was found, with an in-plane bent $\mathrm{CCH}$ acetyleno group, due to rehybridisation of the acetyleno carbon atoms, comparable to the rehybridised bent cyano group in the case of DMABN. In this second paper [2] two local minima were predicted to exist on the potential energy surface of the lowest excited singlet state: one with a twisted dimethylamino group and a second with an in-plane bent cyano (DMABN) or acetyleno (DACET) group. It was concluded [2] that in DMABN the TICT and RICT mechanisms may compete with each other in the ICT reaction. For this reaction in DACET, however, the bending of the acetyleno group was expected to be strongly preferred over the twisting of the dimethylamino group, as the RICT state represents here the global minimum of the potential energy surface [2].

In order to test the prediction that DACET would be dual fluorescent, this molecule was synthesised and its photostationary and time-resolved photophysical properties such as fluorescence decays and quantum yields were studied. The results of these experiments are presented here, showing that neither dual fluorescence nor an ICT reaction is observed with DACET.

\section{Experimental}

DACET was synthesised following a procedure described in the literature [15]. ${ }^{1} \mathrm{H}-\mathrm{NMR}\left(\mathrm{CDCl}_{3}\right): \delta$ $=2.98\left(\mathrm{~s}, 7 \mathrm{H}, 2 \times \mathrm{CH}_{3}+\mathrm{CH}\right), 6.63(\mathrm{~d}, 2 \mathrm{H}, J=$ $8 \mathrm{~Hz}$, arom. $\mathrm{H}), 7.38(\mathrm{~d}, 2 \mathrm{H}, J=8 \mathrm{~Hz}$, arom. $\mathrm{H})$. The melting point was $52-53^{\circ} \mathrm{C}$. DACET was purified by HPLC immediately before the experiments. It was found that this molecule readily undergoes a reaction, probably a polymerisation [16], in the dark as well as under laser excitation. Unless fresh solutions are used, this reaction leads to the appearance of a new red-shifted band between 25000 and 30000 $\mathrm{cm}^{-1}$ in the absorption spectrum. In the fluorescence spectrum of the degraded DACET a new emission appears, red-shifted with respect to its LE band, with a maximum around $20500 \mathrm{~cm}^{-1}$ (diethyl ether). The synthesis of 4-(methylamino)benzonitrile (MABN) has been described previously [12]. MABN and DMABN (Aldrich) were purified by HPLC.

The solvent n-hexane (Merck, Uvasol) was used as received. Diethyl ether (Uvasol) and acetonitrile (Baker, for HPLC) were purified by chromatography 
over alumina and alumina plus active charcoal, respectively, just prior to use. The other solvents, employed in the solvatochromic measurements, were chromatographed over alumina or alumina and active charcoal (proprionitrile). Toluene (Uvasol) was refluxed with potassium. No extraneous fluorescence was found to be present under the prevailing excitation conditions.

The fluorescence spectra were measured by using a quantum-corrected Shimadzu 5000PC spectrophotometer. The fluorescence quantum yields $\Phi$ were determined at $25^{\circ} \mathrm{C}$ by comparison with a quinine sulphate solution (in $1.0 \mathrm{~N} \mathrm{H}_{2} \mathrm{SO}_{4}, \Phi=$ 0.546 [17]) of equal optical density at the excitation wavelength.

The nanosecond and picosecond time-correlated single-photon counting (SPC) data were obtained with systems described previously $[6,12]$. The analysis of the fluorescence decays was carried out by using the method of modulating functions [18].

\section{Results and discussion}

\subsection{Fluorescence spectra}

The fluorescence and absorption spectra of DACET in n-hexane and acetonitrile at $25^{\circ} \mathrm{C}$ are depicted in Fig. 1. The corresponding spectra of DMABN are also presented for comparison. In the fluorescence spectra of both molecules in $\mathbf{n}$-hexane there is no indication of the presence of a dual fluorescence: only an LE emission is observed [19]. In acetonitrile, the DACET fluorescence spectrum likewise consists exclusively of an LE emission band, in clear contrast with the dual fluorescence of predominant CT character $[6,13]$ observed with DMABN in acetonitrile, as shown in the right part of Fig. 1. This absence of dual fluorescence in the case of DACET is confirmed over the entire temperature range accessible with $\mathrm{n}$-hexane and acetonitrile.

\subsection{Absorption spectra}

It is seen from the absorption spectra in n-hexane (Fig. 1) that the energy difference between the first vibrational peak of the lower energy structured ab- sorption and the maximum of the stronger structureless band is larger for DACET $\left(4150 \mathrm{~cm}^{-1}\right)$ than for DMABN $\left(3820 \mathrm{~cm}^{-1}\right)$, see Table 1 . This energy difference is related to the energy gap $\Delta E\left(S_{1}, S_{2}\right)$ between the two lowest excited singlet states [5-7]. Larger values for this gap are found (Table 1) for MABN (4540 $\left.\mathrm{cm}^{-1}\right)$ and especially for 4-aminobenzonitrile $(\mathrm{ABN})\left(5130 \mathrm{~cm}^{-1}\right)$. In the polar solvent acetonitrile the main absorption maximum of DMABN is more strongly red-shifted relative to n-hexane than the structured band, leading to a complete overlap of the last absorption (Fig. 1). With DACET in acetonitrile the weaker band is still clearly visible, due to a smaller red-shift of the strong absorption maximum of DACET as compared with that of DMABN, see Fig. 1. This observation means that in acetonitrile the energy gap $\Delta E\left(S_{1}, S_{2}\right)$ of DACET is considerably larger than that of DMABN.

\subsection{Energies $E\left(S_{1}\right)$ and energy gaps $\Delta E\left(S_{I}, S_{2}\right)$}

Experimental and theoretical data for the energy $E\left(S_{1}\right)$ of the $S_{1}$ state relative to the ground state and the energy gap $\triangle E\left(S_{1}, S_{2}\right)$ of DACET, DMABN, MABN and ABN are listed in Table 1. The experimental energy $E\left(S_{1}\right)$ of DMABN, as an example, decreases due to solvation from $32210 \mathrm{~cm}^{-1}$ in the gas phase $\left(32260 \mathrm{~cm}^{-1}\right.$ under jet conditions [8]) to $31970 \mathrm{~cm}^{-1}$ in perfluoromethylcyclohexane and $31650 \mathrm{~cm}^{-1}$ in the more strongly solvating $\mathrm{n}$-hexane. The energy gap $\Delta E\left(S_{1}, S_{2}\right)$, approximated from the absorption spectra as described in Section 3.2, decreases from $4320 \mathrm{~cm}^{-1}$ in the gas phase to 3820 $\mathrm{cm}^{-1}$ in $\mathrm{n}$-hexane. These data refer to the vertical transition from the $S_{0}$ state, as is also the case for the theoretical data $[1,2]$. The calculated value $[1,2]$ for $E\left(S_{1}\right)$ of DMABN $\left(33310 \mathrm{~cm}^{-1}\right)$ is larger than the experimental energy in the gas phase, whereas for $\Delta E\left(S_{1}, S_{2}\right)\left(3060 \mathrm{~cm}^{-1}\right)$ theory predicts a considerably smaller value than that observed. A similar trend is found for the energy $E\left(S_{1}\right)$ of DACET and $A B N$, while the calculated and experimental energy gaps $\Delta E\left(S_{1}, S_{2}\right)$ of ABN are of comparable magnitude (Table 1). As an important result, the theoretical calculations [1,2] agree with experiment in concluding that the energy gap $\Delta E\left(S_{1}, S_{2}\right)$ is larger for DACET than for DMABN. 


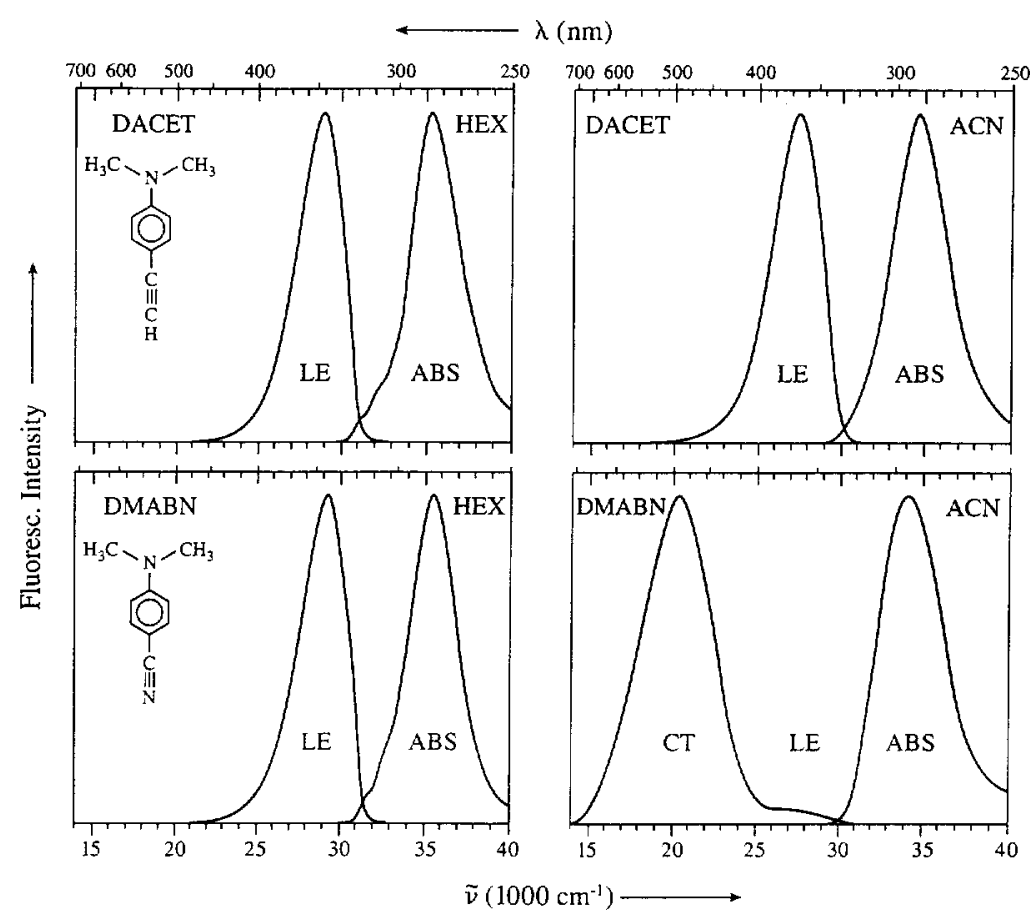

Fig. 1. Fluorescence and absorption spectra of (upper part) 4-(dimethylamino)phenylacetylene (DACET) and (lower part) 4-(dimethylamino)benzonitrile (DMABN) in n-hexane (HEX) and acetonitrile (ACN) at $25^{\circ} \mathrm{C}$. The fluorescence spectra of both molecules in $n$-hexane consist of an emission from a locally excited (LE) state, whereas with DMABN in acetonitrile a dual fluorescence from an LE and a charge transfer (CT) state is observed, in contrast with the single LE emission band of DACET in this solvent.

\subsection{Solvent polarity dependence of fluorescence spectra}

The fluorescence spectrum of DACET was measured at $25^{\circ} \mathrm{C}$ in a variety of solvents of different molecular nature and polarity. The solvent polarity is represented here by the polarity scale $f-f^{\prime}$ (Eq.
(1)), where $\epsilon$ and $n$ are the dielectric constant and the refractive index of the solvent $[21,22]$.

$$
f-f^{\prime}=(\epsilon-1) /(2 \epsilon+1)-\left(n^{2}-1\right) /\left(2 n^{2}+1\right) .
$$

For all solvents studied, only a single emission band

Table 1

Experimental and calculated energies $E\left(S_{1}\right)$ and energy gaps $\Delta E\left(S_{1}, S_{2}\right)$ in $1000 \mathrm{~cm}^{-1}$ of 4-(dimethylamino)phenylacetylene (DACET), 4-(dimethylamino)benzonitrile (DMABN), 4-(methylamino)benzonitrile (MABN) and 4-aminobenzonitrile (ABN)

\begin{tabular}{|c|c|c|c|c|c|c|c|c|}
\hline & \multicolumn{2}{|c|}{ DACET } & \multicolumn{2}{|c|}{ DMABN } & \multicolumn{2}{|c|}{ MABN } & \multicolumn{2}{|l|}{$A B N$} \\
\hline & $E\left(S_{1}\right)$ & $\Delta E\left(S_{1}, S_{2}\right)$ & $E\left(S_{1}\right)$ & $\Delta E\left(S_{1}, S_{2}\right)$ & $E\left(S_{1}\right)$ & $\Delta E\left(S_{1}, S_{2}\right)$ & $E\left(S_{1}\right)$ & $\Delta E\left(S_{1}, S_{2}\right)$ \\
\hline gas phase & - & - & 32.21 & 4.32 & - & - & - & - \\
\hline jet & - & - & 32.26 & - & - & - & 33.47 & - \\
\hline perfluoromethylcyclohexane & 31.42 & 4.36 & 31.97 & 4.05 & 32.46 & 4.85 & $33.24^{\text {a }}$ & $5.61^{a}$ \\
\hline n-hexane & 31.03 & 4.15 & 31.65 & 3.82 & 32.15 & 4.54 & 32.98 & 5.13 \\
\hline theory (Refs. $[1,2]$ ) & 32.83 & 3.87 & 33.31 & 3.06 & - & - & 34.36 & 5.81 \\
\hline
\end{tabular}

\footnotetext{
a In perfluorohexane.
} 
Table 2

Energies $h \nu^{\max }$ of the maximum of the locally excited (LE) fluorescence band of 4-(dimethylamino)phenylacetylene (DACET) and 4-(methylamino)benzonitrile (MABN) and of the maxima of the LE and charge transfer (CT) bands of 4-(di-methylamino)benzonitrile (DMABN) in a series of solvents at $25^{\circ} \mathrm{C}$ spanning the polarity scale $f-f^{\prime}$. For DMABN the CT/LE fluorescence quantum yield ratio $\Phi^{\prime} / \Phi$ is listed

\begin{tabular}{|c|c|c|c|c|c|c|c|c|}
\hline \multirow[t]{3}{*}{ Solvent } & \multirow{3}{*}{$\begin{array}{l}f-f^{\prime} \\
\text { (Eq. (1)) }\end{array}$} & \multirow{3}{*}{$\begin{array}{l}\epsilon^{25} \\
\text { Ref. [21] }\end{array}$} & \multirow{3}{*}{$\begin{array}{l}n_{\mathrm{D}}^{25} \\
\text { Ref. [21] }\end{array}$} & \multirow{3}{*}{$\begin{array}{l}\text { DACET } \\
h \nu^{\max }(\mathrm{LE}) \\
{\left[1000 \mathrm{~cm}^{-1}\right]}\end{array}$} & \multirow{3}{*}{$\begin{array}{l}\text { MABN } \\
h \nu^{\max }(\mathrm{LE}) \\
{\left[1000 \mathrm{~cm}^{-1}\right]}\end{array}$} & \multicolumn{3}{|l|}{ DMABN } \\
\hline & & & & & & $h \nu^{\max }(\mathrm{LE})$ & $h \nu^{\max }(\mathrm{CT})$ & $\Phi^{\prime} / \Phi$ \\
\hline & & & & & & {$\left[1000 \mathrm{~cm}^{-1}\right]$} & {$\left[1000 \mathrm{~cm}^{-1}\right]$} & \\
\hline perfluoromethylcyclohexane (1) & 0.028 & 1.82 & 1.279 & 29.2 & 30.39 & 29.59 & - & 0.0 \\
\hline n-hexane (2) & 0.000 & 1.88 & 1.372 & 28.86 & 30.09 & 29.31 & - & 0.0 \\
\hline toluene (3) & 0.013 & 2.37 & 1.494 & 28.07 & 29.23 & 28.4 & 24.4 & 0.13 \\
\hline di(n-butyl) ether (4) & 0.092 & 3.05 & 1.404 & 28.50 & 29.28 & 28.7 & 25.3 & 0.09 \\
\hline di(n-propyl) ether (5) & 0.116 & 3.39 & 1.388 & 28.46 & 29.24 & 28.7 & 24.8 & 0.12 \\
\hline diethyl ether (6) & 0.161 & 4.27 & 1.361 & 28.38 & 29.15 & 28.4 & 23.9 & 0.29 \\
\hline ethylacetate (7) & 0.200 & 6.02 & 1.370 & 27.84 & 28.73 & 27.9 & 22.1 & 2.8 \\
\hline tetrahydrofuran (8) & 0.208 & 7.39 & 1.405 & 27.90 & 28.58 & 27.8 & 22.2 & 2.5 \\
\hline propionitrile (9) & 0.290 & 27.2 & 1.366 & 27.67 & 28.68 & 27.6 & 21.0 & 13 \\
\hline acetonitrile (10) & 0.306 & 36.0 & 1.342 & 27.41 & 28.64 & (27.6) & 20.3 & 29 \\
\hline n-propanol (11) & 0.274 & 20.3 & 1.385 & 27.98 & 28.60 & 28.1 & 20.6 & 18 \\
\hline ethanol (12) & 0.289 & 24.6 & 1.360 & 27.84 & 28.59 & 28.0 & 20.3 & 19 \\
\hline methanol (13) & 0.309 & 32.7 & 1.326 & 27.68 & 28.61 & $(27.8)$ & 19.7 & 22 \\
\hline
\end{tabular}

(see Fig. 1 and Section 3.1) appears in the fluorescence spectrum. The energies of the band maximum $h \nu^{\max }(\mathrm{LE})$ are presented in Table 2 , together with those of the LE emission band of MABN and the CT and LE band maxima of DMABN. With DMABN in acetonitrile at $25^{\circ} \mathrm{C}$ the relatively small band around $27000 \mathrm{~cm}^{-1}$ (see Fig. 1) contains an important contribution from degradation products [23].

The red-shift with increasing solvent polarity experienced by the fluorescence band of DACET is of approximately the same magnitude as that found for the LE band of MABN and DMABN. The data for $h \nu^{\text {max }}$ (LE) are plotted against $f-f^{\prime}$ in Fig. 2, clearly demonstrating the similarity of the photostationary spectral behaviour of DACET and MABN. As it has been established that in the case of MABN an ICT reaction does not take place in the excited singlet state $[5,12,19]$, the same conclusion is drawn here for DACET.

The solvent polarity dependence of the fluorescence spectrum of DACET is completely different from that of DMABN, however (Fig. 2 and Table 2). With the dual fluorescent DMABN, the energy $h \nu^{\max }(\mathrm{CT})$ of the maximum of the CT emission band strongly decreases with increasing solvent polarity $[12,19]$, see Fig. 2. This behaviour is in accord with the considerably larger dipole moment $\mu_{\mathrm{e}}(\mathrm{CT})$ $\simeq 17 \mathrm{D}$ of the CT state as compared to the dipole moment $\mu_{\mathrm{e}}(\mathrm{LE}) \simeq 10 \mathrm{D}$ of the LE state, as determined from time resolved microwave conductivity (TRMC) experiments [19] and also from solvatochromic measurements [24].
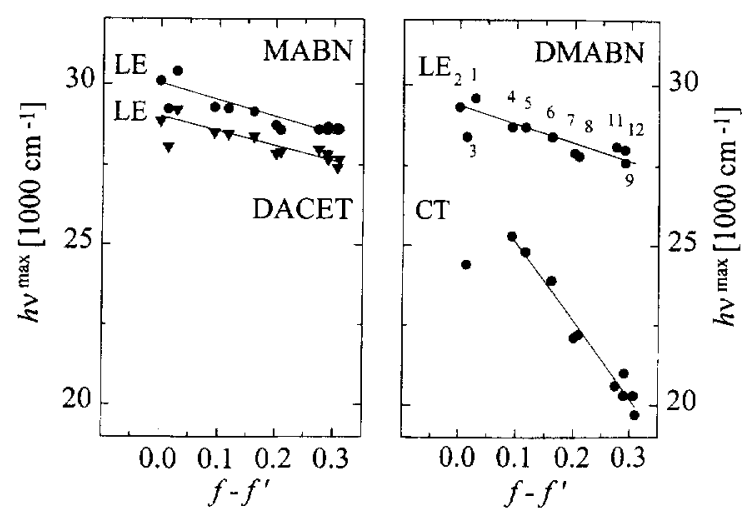

Fig. 2. Solvatochromic plots of the energies $h \nu^{\max }$ of the maxima of the locally excited (LE) fluorescence bands of 4(methylamino)benzonitrile (MABN), 4-(dimethylamino)phenylacetylene (DACET) and 4-(dimethylamino)benzonitrile (DMABN) and also of the charge transfer (CT) emission band of DMABN. The horizontal axis is the polarity scale $f-f^{\prime}$, see text (Eq. (3)) and Table 2. The numbering of the solvents is given in Table 2 . The data for the solvent toluene (3) are not taken into account in the linear fits. 
From the results presented here and in Section 3.1 it is therefore concluded that an inspection of the photostationary fluorescence spectra of DACET un-

DACET
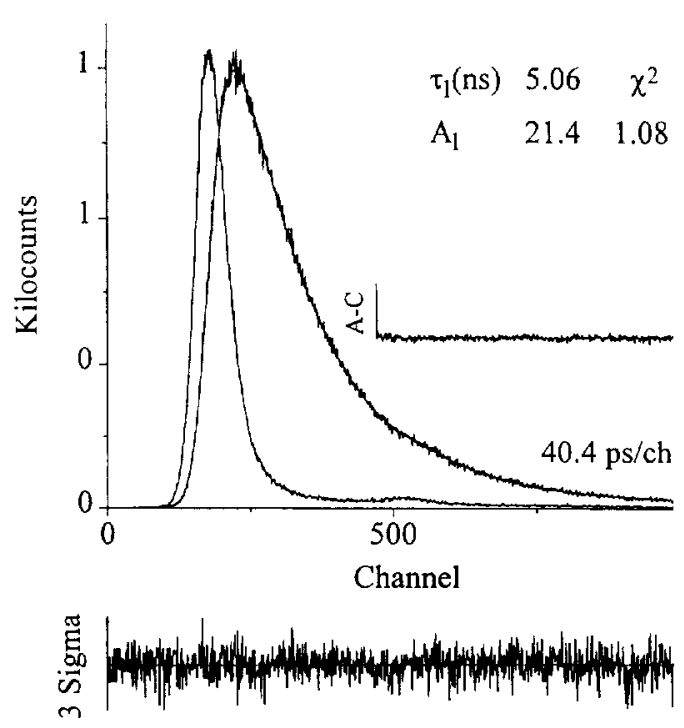

acetonitrile
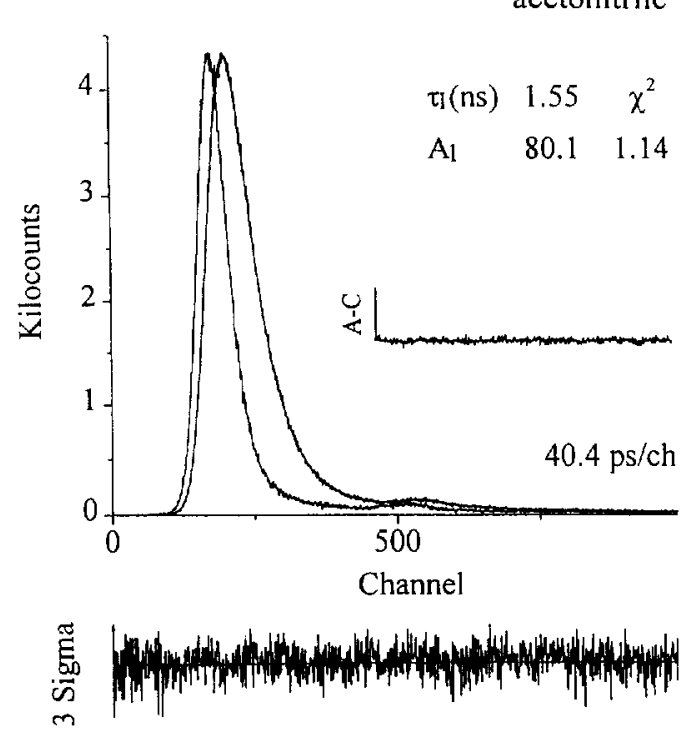

Fig. 3. Fluorescence response functions of the LE emission of 4-(dimethylamino)phenylacetylene (DACET) in (top) n-hexane and (bottom) acetonitrile at $25^{\circ} \mathrm{C}$. The decay times $\left(\tau_{1}\right)$ and their preexponential factors $A_{1}$ are given. The decays were obtained by using nanosecond flash lamp excitation at $296 \mathrm{~nm}$, with $40.4 \mathrm{ps}$ per channel. The weighted deviations, expressed as $3 \sigma$ (expected deviations) and the autocorrelation functions $A-C$ are indicated. der a large variety of experimental conditions does not reveal the occurrence of an ICT reaction with this molecule in the singlet excited state, contrary to the prediction of Sobolewski and Domcke [1,2].

\subsection{Time-resolved measurements. Nanosecond and picosecond time resolution}

The fluorescence decay of DACET in n-hexane is purely single exponential, as determined from nanosecond SPC measurements, with a lifetime of $5.06 \mathrm{~ns}$ at $25{ }^{\circ} \mathrm{C}$ (Fig. 3) and $5.67 \mathrm{~ns}$ at $-70^{\circ} \mathrm{C}$.

With DACET in acetonitrile similar single exponential fluorescence decays are obtained over the entire temperature range between -44 and $75^{\circ} \mathrm{C}$. The fluorescence decay time $\tau$ of DACET in acetonitrile decreases from $4.44 \mathrm{~ns}$ at $-44^{\circ} \mathrm{C}$ to $0.63 \mathrm{~ns}$ at $75^{\circ} \mathrm{C}$. As the fluorescence quantum yield $\Phi$ similarly decreases by a factor of around 7 and an additional picosecond decay time does not appear, it is concluded that the decrease in $\tau$ with increasing temperature is not caused by an ICT reaction (see Section 3.6).

The decays measured at $25^{\circ} \mathrm{C}$ are depicted in Figs. 3 and 4 . The nanosecond SPC experiments, with a time resolution of $40 \mathrm{ps}$ per channel, result in a fluorescence decay time $\tau_{1}$ of $1.55 \mathrm{~ns}$ (Fig. 3). From the SPC experiments carried out with the picosecond laser system (Fig. 4), however, a double exponential fluorescence decay is obtained, with the decay times $\tau_{1}=1.55 \mathrm{~ns}$ and $\tau_{p}=4.16 \mathrm{~ns}$. The additional time $\tau_{p}$ appearing in these SPC measurements is attributed to a photoproduct of DACET (see Experimental), which does not appear under the very mild excitation conditions of the nanosecond SPC experiments (flashlamp excitation).

The occurrence of an ICT reaction in electron donor/acceptor-substituted benzenes generally is accompanied by the appearance of a second decay time in the picosecond domain, especially at low temperatures, where the thermally activated back reaction $\mathrm{CT} \rightarrow \mathrm{LE}$ is slowed down with respect to the reciprocal CT lifetime [12]. The CT/LE fluorescence quantum yield ratio is given by Eq. (2)

$$
\frac{\Phi^{\prime}(\mathrm{CT})}{\Phi(\mathrm{LE})}=\frac{k_{f}^{\prime}}{k_{f}} \cdot \frac{k_{a}}{\left(k_{d}+1 / \tau_{0}^{\prime}\right)},
$$

where $k_{f}^{\prime} / k_{f}$ is the ratio of the radiative rates for the 
CT and LE emissions, $k_{a}$ and $k_{d}$ are the forward and backward ICT reaction rates and $\tau_{0}^{\prime}$ is the lifetime of the CT state [12]. Whether an emissive CT state can be detected obviously depends on the ratio $k_{a} /\left(k_{d}\right.$ $\left.+1 / \tau_{0}^{\prime}\right) . \Phi^{\prime}(\mathrm{CT}) / \Phi(\mathrm{LE})$ will be close to zero, i.e., a CT emission cannot easily be observed, when $k_{a}$ is very small (possibly due to a large activation barrier for the LE $\rightarrow$ CT reaction) or when $k_{d}$ is very large (a small value for the CT stabilisation enthalpy $-\Delta H[12])$. The optimal temperature range to detect a CT emission depends on the relative values of $k_{d}$ and $1 / \tau_{0}^{\prime}$ : low temperatures when $k_{d} \gg 1 / \tau_{0}^{\prime}$ (small $-\Delta H$, as with DMABN in toluene [12]) and high temperatures when $k_{d} \ll 1 / \tau_{0}^{\prime}$.

The absence of an additional picosecond decay time in the time-resolved fluorescence measurements with a resolution of around 5 ps presented here, therefore supports our previous conclusion based on photostationary experiments that an ICT reaction does not take place with DACET in n-hexane or in acetonitrile, neither in the low- or high-temperature range [12].

\subsection{Fluorescence quantum yields and decay times.}

\section{Radiative rate constants}

The fluorescence quantum yields $\Phi$ and decay times $\tau$ of DACET were determined in three solvents of different polarity: n-hexane, diethyl ether and acetonitrile (Table 3). The fluorescence quantum yield in $\mathbf{n}$-hexane is relatively large, with a value of 0.28 . For DMABN and MABN, in cyclohexane, similar quantum yields have been measured: 0.16 and 0.12 , respectively [19]. With the 4-aminobenzonitriles, such $\Phi$ values mean that no ICT takes place. In the case of DMABN practically no CT emission occurs in alkane solvents [6,19], whereas with MABN only LE fluorescence is found in all solvents, irrespective of solvent polarity [5,12].

The time $\tau$ derived from the single exponential fluorescence decay of DACET decreases in the same manner from n-hexane to acetonitrile as the quantum yield $\Phi$, see Table 3 . This means that the radiative rate constant $k_{f}(=\Phi / \tau)$ remains practically constant for the three solvents, especially when its value is corrected for the differences in solvent refractive index $n$, via the relation $k_{f} / n^{2}$ [25]. It is therefore concluded that the molecular nature of the singlet
DACET acetonitrile
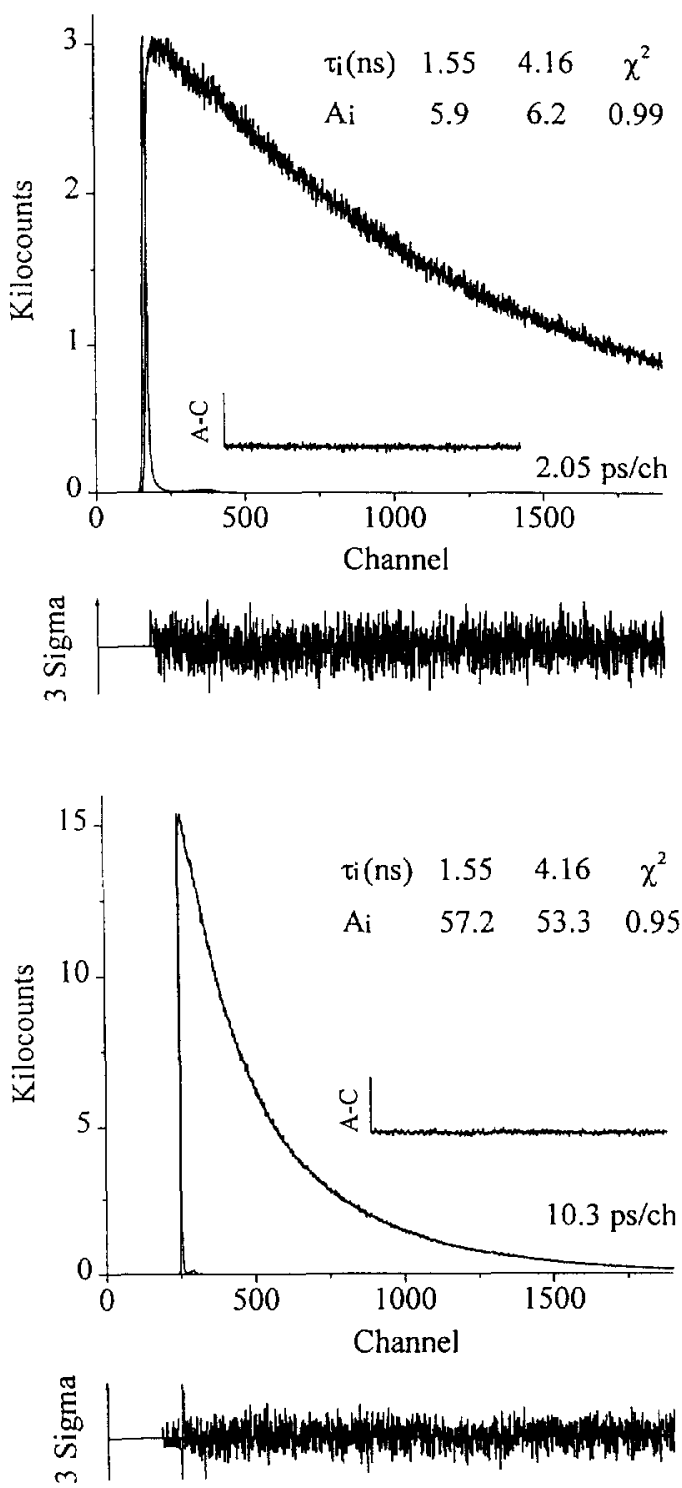

Fig. 4. Fluorescence response functions of the LE emission of 4-(dimethylamino)phenylacetylene (DACET) in acetonitrile at $25^{\circ} \mathrm{C}$. The decay times $\left(\tau_{i}\right)$ and their preexponential factors $A_{i}$ are given. The decays were obtained by using picosecond laser excitation at $296 \mathrm{~nm}$, with (top) $2.05 \mathrm{ps} /$ channel and (bottom) 10.3 ps/channel. The decay time $\tau_{2}$ of $4.16 \mathrm{~ns}$ is due to photochemical and dark degradation of DACET. It does not appear in decays obtained with flash lamp excitation, see Fig. 3 and text. The weighted deviations, expressed as $3 \sigma$ (expected deviations) and the autocorrelation functions $A-C$ are indicated. 
Table 3

Fluorescence quantum yields $\Phi$, fluorescence decay times $\tau$ and radiative rate constant $k_{f}$ of 4-(dimethylamino)phenylacetylene (DACET) in three solvents of different polarity at $25^{\circ} \mathrm{C}$

\begin{tabular}{lllllll}
\hline Solvent & $\Phi$ & $\epsilon^{25}$ & $\tau(\mathrm{ns})$ & $k_{f}\left(10^{7} \mathrm{~s}^{-1}\right)$ & $n_{\mathrm{D}}^{25}$ & $k_{f} / n^{2}\left(10^{7} \mathrm{~s}^{-1}\right)$ \\
\hline n-hexane (2) & 0.28 & 1.88 & 5.06 & 5.53 & 1.372 & 2.94 \\
diethyl ether (6) & 0.22 & 4.27 & 4.04 & 5.45 & 1.361 & 2.94 \\
acetonitrile (10) & 0.08 & 36.0 & 1.55 & 5.16 & 1.342 & 2.87 \\
\hline
\end{tabular}

excited state of DACET is independent of solvent polarity, in accordance with our conclusion that with DACET neither in n-hexane nor in acetonitrile an ICT reaction occurs in the singlet excited state. The decrease in $\Phi$ and $\tau$ from n-hexane to acetonitrile is hence due to nonradiative processes: intersystem crossing and/or internal conversion.

\subsection{Solvatochromic data. $L E$ dipole moment of DACET}

In the calculations of Sobolewski and Domcke $[1,2]$ the magnitude of the dipole moment plays an important role in the identification of the molecular nature of the various excited states of DACET and DMABN. In this section the dipole moment of the LE state of DACET is therefore discussed. Its determination is based on the excited state dipole moments of MABN (LE) and DMABN (LE and CT), which have been measured by employing the TRMC method [19], see Table 4.
The energies $h \nu^{\max }$ (LE) of the emission maxima of DACET are plotted in Fig. 5 against those of MABN as well as those of DMABN (Table 2). From the slope of these plots, the dipole moment of the LE state of DACET can be determined by using $[20,24,26]$

$$
\begin{aligned}
\tilde{\nu}_{\text {flu }}= & \frac{-1}{4 \pi \epsilon_{0}} \frac{2}{h c \rho^{3}} \mu_{\mathrm{e}}^{\mathrm{LE}}\left(\mu_{\mathrm{e}}^{\mathrm{LE}}-\mu_{\mathrm{g}}^{\mathrm{FC}}\right)\left(f-f^{\prime}\right) \\
& + \text { const. }
\end{aligned}
$$

In Eq. (3), $\rho$ is the equivalent spherical radius of the solute (Onsager radius) $[20,24]$ and $\epsilon_{0}$ is the vacuum permittivity of the solvent. $\mu_{\mathrm{e}}^{\mathrm{LE}}$ is the dipole moment of the relaxed LE state. It is assumed here that the dipole moment $\mu_{\mathrm{g}}^{\mathrm{FC}}$ of the Franck-Condon (FC) ground state reached upon emission from this LE state is identical with the dipole moment $\mu_{\mathrm{g}}$ of the equilibrated ground state $S_{0}$ [20].

The slope of the plots in Fig. 5 is equal to the ratio of the expression $\mu_{\mathrm{e}}^{\mathrm{LE}}\left(\mu_{\mathrm{e}}^{\mathrm{LE}}-\mu_{\mathrm{g}}\right) / \rho^{3}$ (Eq. (3))
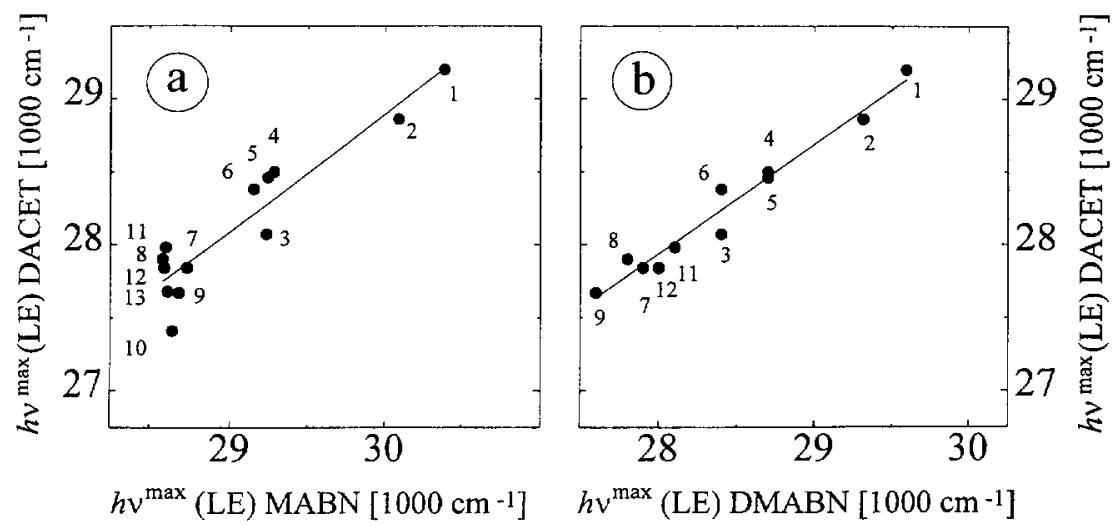

Fig. 5. Plots of the energies $h \nu^{\max }$ (LE) of the maxima of the locally excited (LE) fluorescence bands of 4-(dimethylamino)phenylacetylene (DACET) against those of (a) 4-(methylamino)benzonitrile (MABN), and (b) 4-(dimethylamino)benzonitrile (DMABN) in a series of solvents of different polarity, see Table 2 and Fig. 2. The numbering of the solvents is given in Table 2. 
Table 4

Dipole moments $\mu_{\mathrm{g}}\left(\mathrm{S}_{0}\right)$ of the ground state, $\mu_{\mathrm{e}}(\mathrm{LE})$ of the locally excited state (LE) and $\mu_{\mathrm{e}}(\mathrm{CT})$ of the charge transfer (CT) state of 4-(dimethylamino)phenylacetylene (DACET), 4-aminobenzonitrile ( $\mathrm{ABN}$ ), 4-(methylamino)benzonitrile (MABN) and 4-(dimethylamino)benzonitrile (DMABN)

\begin{tabular}{|c|c|c|c|}
\hline & $\mu_{\mathrm{g}}\left(\mathrm{S}_{0}\right)[\mathrm{D}]$ & $\mu_{\mathrm{e}}(\mathrm{LE})[\mathrm{D}]$ & $\mu_{\mathrm{e}}(\mathrm{CT})[\mathrm{D}]$ \\
\hline DACET & $2.7^{\mathrm{a}}$ & 7 & - \\
\hline DACET (theory) a & 2.7 & $(2.9)^{b}$ & $10.6($ TICT $) / 8.7($ RICT $)$ \\
\hline DMABN & 6.6 & 10 & 17 \\
\hline DMABN (theory) a & 6.6 & 6.9 & 14.7 (TICT) / 16.4 (RICT) \\
\hline MABN c & 6.6 & 9.9 & - \\
\hline $\mathrm{ABN}{ }^{\circ}$ & 6.6 & 8.3 & - \\
\hline $\mathrm{ABN}$ (theory) a & 6.2 & 6.1 & - \\
\hline
\end{tabular}

a From Ref. [2]. See text for the meaning of TICT and RICT.

b From Ref. [2]: lowest excited singlet state $S_{1}\left(\mathrm{~B}_{2}\right)$.

c From Ref. [19].

for DACET and MABN (Fig. 5a) or DMABN (Fig. $5 b)$. From the values of these slopes, DACET $/ \mathrm{MABN}=0.81$ and DACET/DMABN $=0.75$, together with the data for $\mu_{\mathrm{g}}$ and $\mu_{\mathrm{e}}^{\mathrm{LE}}(\mathrm{MABN}, \mathrm{DMABN})$ from Table 4 and the difference in length of the molecules [27], a $\mu_{\mathrm{e}}^{\mathrm{LE}}$ dipole moment of $7 \mathrm{D}$ is calculated for DACET from both plots in Fig. 5, see Table 4.

The dipole moment $\mu_{\mathrm{e}}^{\mathrm{LE}}$ of DACET can in principle also be determined from the slope $\mu_{\mathrm{e}}^{\mathrm{LE}}\left(\mu_{\mathrm{e}}^{\mathrm{LE}}-\right.$ $\left.\mu_{\mathrm{g}}\right) / \rho^{3}$ (Eq. (3)) of the plot of $h \nu^{\max }$ (LE) of DACET against $f-f^{\prime}$ in Fig. 2. Using this method, the same value for $\mu_{\mathrm{e}}^{\mathrm{LE}}$ of $7 \mathrm{D}$ is obtained. In this approach, the radius $\rho$ of DACET is calculated (Eq. (3)) from the corresponding slope for DMABN and MABN in Fig. 2 and the known LE and $S_{0}$ dipole moments of MABN and DMABN [19], taking into account the difference in molecular size as discussed above. In the more accurate method based on Fig. 5, however, the specific solute/solvent interactions leading to the scatter of the data points in Fig. 2, such as those in toluene, are mutually compensated.

The dipole moment of $7 \mathrm{D}$ determined here for the $S_{1}$ state of DACET is lower than that calculated for the RICT $(8.7 \mathrm{D})$ as well as the TICT $(10.6 \mathrm{D})$ state of this molecule $[1,2]$, see Table 4 .

\section{Discussion and conclusions}

The electron donor/acceptor-substituted benzene derivative DACET does not undergo an ICT reaction in the singlet excited state, contrary to the prediction made by Sobolewski and Domcke on the basis of $a b$ initio electronic structure calculations [2]. The fluorescence from the $S_{1}$ state of DACET originates from an LE state independent of solvent polarity, as deduced from the lack of dual fluorescence and the single exponential fluorescence decays observed with this molecule. The a priori possibility that the RICT (or TICT) state of DACET is in fact close in energy to the LE state but cannot be reached because of a prohibitive energy barrier on the reaction pathway to the CT state is not experimentally verifiable.

The absence of dual emission in the case of DACET is in line with our hypothesis $[5-7,14]$ that a relatively small energy gap $\Delta E\left(S_{1}, S_{2}\right)$ between the two lowest singlet excited states, leading to vibronic coupling of $S_{1}$ and $S_{2}$, is a requirement for the occurrence of an ICT reaction in 4-aminobenzonitriles. As discussed in Sections 3.2 and 3.3, the energy gap $\Delta E\left(S_{1}, S_{2}\right)$ of DACET is clearly larger than that of DMABN in n-hexane and remains relatively large in acetonitrile, due to the smaller difference between the dipole moments of the $S_{1}$ and $S_{2}$ states in the case of DACET. In our 'planar intramolecular charge tranfer' (PICT) model [14] it is postulated that the final CT state of DMABN is planar, based on the finding [13] that in this state the dimethylamino group is strongly coupled with the benzonitrile moiety.

A second important factor in causing the absence of dual fluorescence in the case of DACET is the much weaker electron acceptor strength of the 
acetyleno substituent as compared with the cyano group, which can be deduced from their respective influence on spectral properties $[28,29]$. With DMABN and related dual fluorescent 4-aminobenzonitriles the combination of the amino and cyano substituents just brings the $S_{1}$ and $S_{2}$ levels in proximity. Replacement of the dimethylamino group by the weaker electron donor substituent $\mathrm{OCH}_{3}$ $[28,29]$, as an example, leads to the disappearance of dual fluorescence and an increase in $\Delta E\left(S_{1}, S_{2}\right)$ relative to DMABN is observed [30,31].

The results of this paper indicate that excited state calculations at the present state of development do not always lead to conclusions that are immediately relevant in discussions of the detailed dynamics of excited state processes such as ICT and dual fluorescence in molecules of the size and complexity of DMABN and DACET. This might especially be the case with DMABN and related systems having a relatively small energy gap $\Delta E\left(S_{1}, S_{2}\right)$, as the accuracy of calculated excited state energies is generally not better than around $0.2 \mathrm{eV}\left(1600 \mathrm{~cm}^{-1}\right)[2,32]$. see Table 1.

Our conclusion should be seen in the light of the striking difference between the findings of three recent theoretical approaches $[1,2,9,10]$ concerning the molecular configuration of the CT state of DMABN. In calculations using the CIS method, Scholes et al. [10] presented evidence against the importance of a RICT state in DMABN. They supported their claim by indicating that the experimentally observed frequency decrease [33] for the stretching of the cyano group in the CT state of DMABN is not found in their calculations of the state with a RICT structure.

\section{Acknowledgements}

Many thanks are due to Dr. A.L. Sobolewski for discussions on the details of his structure calculations. The continuing support from Dr. W. Kühnle in the purification and characterisation of the molecules used in our investigations is gratefully acknowledged. Dr. Yu. V. Il'ichev is thanked for measuring a number of picosecond fluorescence decays. The present studies were supported by the Volkswagen-
Foundation (Project 'Intra- and Intermolecular Electron Transfer').

\section{References}

[1] A.L. Sobolewski, W. Domcke, Chem. Phys. Lett. 250 (1996) 428.

[2] A.L. Sobolewski, W. Domcke, Chem. Phys. Lett. 259 (1996) 119.

[3] Z.R. Grabowski, K. Rotkiewicz, A. Siemiarczuk, D.J. Cowley, W. Baumann, Nouv, J. Chim. 3 (1979) 443.

[4] E. Lippert, W. Rettig, V. Bonačič-Koutecký, F. Heisel, J.A. Miehé, Adv. Chem. Phys. 68 (1987) 1.

[5] K.A. Zachariasse, Th. von der Haar, A. Hebecker, U. Leinhos, W. Kühnle, Pure \& Appl. Chem. 65 (1993) 1745.

[6] K.A. Zachariasse, M. Grobys, Th. von der Haar, A. Hebecker, Yu.V. Il'ichev, Y.-B. Jiang, O. Morawski, W. Kühnle, J. Photochem. Photobiol. A: Chem. 102 (1996) 59.

[7] Th. von der Haar, A. Hebecker, Yu. Il'ichev, W. Kühnle, K.A. Zachariasse, Am. Inst. Phys, Conf. Proc. 364 (1996) 295.

[8] R. Howell, D. Phillips, H. Petek, K. Yoshihara, Chem. Phys. 188 (1994) 303.

[9] L. Serrano-Andrés, M. Merchan, B.O. Roos, R. Lindh, J. Am. Chem. Soc. 117 (1995) 3189.

[10] G.D. Scholes, D. Phillips, I.R. Gould, Chem. Phys. Lett. 266 (1997) 521.

[11] R.J. Visser, C.A.G.O. Varma, J. Chem. Soc. Faraday Trans. $276(1980) 453$.

[12] U. Leinhos, W. Kühnle, K.A. Zachariasse, J. Phys. Chem. 95 (1991) 2013.

[13] Th. von der Haar, A. Hebecker, Yu. Il'ichev, Y.-B. Jiang, W. Kühnle, K.A. Zachariasse, Rec. Trav. Chim. Pays-Bas, 114 (1995) 430.

[14] K.A. Zachariasse, Th. von der Haar, A. Hebecker, U. Leinhos, W. Kühnle, J. Photochem. Photobiol. A: Chem. 105 (1997), in press.

[15] S. Akiyama, S. Nakatsuji, K. Yoshida, K. Nakashima, T. Hagiwara, H. Tsuruta, T. Yoshida, Bull. Chem. Soc. Japan 56 (1983) 361.

[16] A. Janoušová, M.J. Beneš, M. Janić, J. Peška, Coll. Czech. Chem. Commun. 39 (1974) 1858.

[17] J.N. Demas, G.A. Crosby, J. Phys. Chem. 75 (1971) 991.

[18] G. Striker, in: Deconvolution and Reconvolution of Analytical Signals, ed. M. Bouchy (University Press, Nancy, 1982), p. 329.

[19] W. Schuddeboom, S.A. Jonker, J.M. Warman, U. Leinhos, W. Kühnle, K.A. Zachariasse, J. Phys. Chem. 96 (1992) 10809.

[20] Yu.V. Il'ichev, W. Kühnle, K.A. Zachariasse, Chem. Phys. 211 (1996) 441

[21] Landolt-Börnstein, Numerical data and functional relationships in science and technology, New series, ed. O. Madelung, Group IV, Vol. 6 (Springer, Berlin, 1991). 
[22] Landolt-Börnstein, Numerical data and functional relationships in science and technology, New series, ed. M.D. Lechner, Group III, Vol. 38B (Springer, Berlin, 1996).

[23] P. Geggier, K.A. Zachariasse, unpublished results.

[24] W. Baumann, H. Bischof, J.-C. Fröhling, C. Brittinger, W. Rettig, K. Rotkiewicz, J. Photochem. Photobiol. A: Chem. 64 (1992) 49.

[25] J.B. Birks, Photophysics of aromatic molecules (Wiley, London, 1970).

[26] W. Liptay, in: Excited states, Vol. 1, ed. E.C. Lim (Academic Press, New York, 1974) p. 129.

[27] J.J. Gajewski, K.E. Gilbert, J. McKelvey, in: Advances in molecular modeling, Vol. II, ed. D. Liotta (JAI Press, Greenwich, CN, 1990), p. 65.

[28] N.S. Isaacs, Physical organic chemistry, 2nd ed. (Longman, Harlow, 1995) ch. 4.
[29] J.N. Murrell, The theory of the electronic spectra of organic molecules (Methuen, London, 1963).

[30] E. Lippert, W. Lüder, H. Boos, in: Advances in molecular spectroscopy: European conference on molecular spectroscopy, Bologna (1959), ed. A. Mangini (Pergamon Press, Oxford, 1962), p. 443.

[31] E. Lippert, W. Lüder, F. Moll, H. Nagele, H. Boos, H. Prigge, I. Siebold-Blankenstein, Angew. Chem. 73 (1961) 695.

[32] B.O. Roos, K. Andersson, M.P. Fülscher, Chem. Phys. Lett. 192 (1992) 5.

[33] M. Hashimoto, H. Hamaguchi, J. Phys. Chem. 99 (1995) 7875. 